\title{
DISCINESIAS INDUZIDAS POR LEVODOPA EM 176 PACIENTES COM DOENÇA DE PARKINSON
}

\author{
MARIA SHEILA G. ROCHA*, LUIZ AUGUSTO F. ANDRADE**, \\ HENRIQUE B. FERRAZ ${ }^{\star \star *}$, VANDERCI BORGES*
}

\begin{abstract}
RESUMO - A ocorrência de discinesias dificulta consideravelmente o manuseio terapêutico dos pacientes parkinsonianos tratados com levodopa. Estudamos as características clínicas das discinesias em 176 pacientes com diagnóstico de doença de Parkinson e tratados com levodopa. As discinesias ocorreram, em média, após 6,2 anos de duraçāo da doença e após 4,2 anos de tratamento com levodopa. A maioria dos pacientes (90\%) achava-se nos estágios II e III de Hoehn \& Yahr por ocasião do início das discinesias. As discinesias mais frequentes foram as de "pico de dose" e "contínua". Movimento do tipo distônico ocorreu em $40 \%$ dos casos e predominou nas discinesias de "fim de dose" e "bifásica". Distonia matinal correspondeu a $35 \%$ dos casos de distonia. Movimentos coreiformes se manifestaram de forma generalizada em 43,2\% dos casos. Movimentos distônicos predominaram nos membros inferiores. A discinesia, quando unilateral, ocorreu mais frequentemente no hemicorpo mais comprometido pela doença de Parkinson. A discinesia orofacial, quando isolada, foi mais frequente nos pacientes mais idosos.
\end{abstract}

PALAVRAS-CHAVE: doença de Parkinson, discinesias, levodopa.

\section{Levodopa-induced dyskinesias in 176 parkisonian patients}

SUMMARY - Dyskinesias are frequently observed in parkinsonian patients during levodopa treatment. The occurrence of these movement disorders usually makes the therapeutic management of the patients very difficult. The clinical characteristics of 176 patients with dyskinesias were retrospectively studied. Dyskinesias occurred, on average, after 6,2 years of duration of Parkinson's disease and after 4.2 years on treatment with levodopa. Patients were more likely to have dyskinesias during more advanced stages (measured by Hoehn and Yahr scale). Peak of dose and square wave were the types of dyskinesia more frequently described and were associated with choreic movements in most cases. Dystonia occurred in $40 \%$ of the cases and was predominant in end of dose and diphasic dyskinesias. Thirty-five percent of dystonia cases presented as "early moming dystonia". Chorea was the most frequent involuntary movement and mostly generalized. Dystonia was most commonly described in lower limbs. Orofacial dyskinesia, when occurred alone, was more frequently seen in old rather than young patients. When dyskinesia was unilateral it was more likely to occur in the side where Parkinson's disease was more severe.

KEY WORDS: Parkinson's disease, dyskinesias, levodopa.

Os primeiros relatos da ocorrência dos movimentos involuntários como complicação do tratamento de pacientes parkinsonianos com o precursor da dopamina foram feitos por Cotzias e seus colaboradores. ${ }^{7}$ Com a difusão do uso da levodopa no tratamento da doença de Parkinson (DP), vários outros relatos sobre a ocorrência das discinesias como complicaçāo do tratamento com a

Setor de Investigação em Moléstias Extrapiramidais da Disciplina de Neurologia Clínica Escola Paulista de Medicina (EPM), Universidade Federal de Sāo Paulo, Sāo Paulo: * Pós-graduanda em Neurologia ** Professor Livre Docente, Chefe do Setor. ${ }^{* * *}$ Doutor em Neurologia, Médico do Setor. Aceite: 1-junho-1995. 
levodopa foram surgindo na literatura $a^{2,39,12,18,23,28,30}$. As discinesias passaram a representar um dos principais fatores limitantes da terapêutica com a levodopa, impossibilitando a administraçāo de doses adequadas da droga. As discinesias associadas com o uso da levodopa podem se manifestar na forma de movimentos coreiformes, distonia, atetose, tiques e mioclonias. Logo que aparecem, as discinesias habitualmente atingem a musculatura orobucolingual e podem não apresentar uma relação nítida com a administração das diversas doses de levodopa durante o dia. Com a progressāo da doença e do tratamento, as discinesias podem se tomar mais graves e atingir outras regiões do corpo ${ }^{21,30}$.

O objetivo principal deste estudo é analisar as características clínicas destas discinesias numa amostra de pacientes parkinsonianos tratados com levodopa.

\section{PACIENTES E MÉTODOS}

Foram revisados 1285 prontuários de pacientes com parkinsonismo atendidos pelo Setor de Investigação em Moléstias Extrapiramidais da EPM/UFSP, no período de janeiro-1978 a dezembro-1992. Foram excluídos da ańlise os prontuários dos pacientes com quadro de parkinsonismo associado a outras patologias degenerativas do sistema nervosos central (SNC), secundário ao uso de drogas, doença vascular cerebral e outras lesōes do SNC. Foram incluídos 176 prontuários de pacientes que preenchiam os critérios delineados para o estudo, ou seja, pacientes com parkinsonismo idiopático em tratamento com levodopa e que apresentaram movimentos involuntários decorrente do tratamento.

Foram analisados os seguintes itens: 1) sexo; 2) idade de início dos sinais ou sintomas parkinsonianos; 3) forma clínica da doença no aparecimento das discinesias; 4) lado predominante dos sinais ou sintomas parkinsonianos; 5) tempo de doença até o início das discinesias; 6) tempo de uso de levodopa; 7) tempo de uso de levodopa até o início da discinesia; 8) dose inicial da levodopa; 9) dose da levodopa no momento do início das discinesias; 10) presença concomitante de flutuação motora (deterioração de fim de dose, acinesia matinal e "on-off"); 11) determinação do estágio da DP no início do tratamento com a levodopa (critérios de Hoehn \& Yahr); 12) determinação do estágio da DP por ocasião do aparecimento das discinesias; 13) tipo de movimento discinético (coréia, distonia, coreodistonia e discinesia orofacial); 14) tipo de discinesia (pico de dose, contínua, bifásica e fim de dose).

\section{RESULTADOS}

Dos 176 pacientes estudados, 67 (38\%) são do sexo feminino e $109(62 \%)$ do sexo masculino, com idade de início da DP variando de 13 a 80 anos (média de 52,5). O tempo de duração da DP até o inicio das discinesias variou de 0,5 a 24 anos, com média de tempo de 6,2 anos. O tempo de uso de levodopa até o momento do aparecimento das discinesias variou de 0,5 a 15 anos, com média de 4,2 anos.

A maioria dos pacientes (85\%) desenvolveu a discinesia fazendo uso de levodopa na dose diária de até $800 \mathrm{mg}$. Na amostra como um todo, a dose média diária foi $614,4 \mathrm{mg}$.

Em relação ao estágio da DP (critérios de Hoehn \& Yahr) no início das discinesias, observamos que os pacientes desenvolveram as discinesias com maior frequência quando se achavam nos estágios II $(39,5 \%)$ e III $(50,5 \%)$.

Os pacientes que iniciaram o tratamento com levodopa durante o estágio I desenvolveram a discinesia em média 5 anos após a introdução da levodopa. Aqueles que iniciaram o tratamento no estágio II levaram em média 4,5 anos para apresentar a discinesia. Introduzindo o tratamento com levodopa quando eles já se encontravam no estágio III, as discinesias surgiram, em média, 2 anos depois. Esta diferença é significante segundo a análise de variância por postos de Kruskal-Wallis (H calc= 17,99; $\mathrm{H}$ crítico $=5,99)$.

Da amostra de 176 pacientes parkinsonianos com discinesias, 104 apresentavam flutuações motoras associadas às discinesias. A maioria destes pacientes $(65,5 \%)$ iniciou o quadro de discinesias no mesmo período do início das flutuações. Do restante, $21,1 \%$ iniciaram as discinesias após o início das flutuaçōes e $15,4 \%$ antes do aparecimento destas. Houve predomínio da flutuaçāo motora 
Tabela 1. Frequência dos tipos de discinesias apresentadas pelos pacientes parkinsonianos tratados com levodopa.

\begin{tabular}{ccc}
\hline Tipo de discinesia & Frequência absoluta(n) & Frequência relativa (\%) \\
\hline Pico de dose & 79 & 40,9 \\
Contínua & 68 & 35,3 \\
Fìm de dose & 28 & 14,5 \\
Bifásica & 12 & 6,2 \\
Não classificadas & 6 & 3,1 \\
Total & 193 & 100 \\
\hline
\end{tabular}

(n) superior ao total da amostra devido a sobreposição de eventos.

do tipo "deterioraçāo de fim de dose" (75,9\%), seguindo-se da associação dos tipos "deterioração de fim de dose" com o tipo "on-off" (16,4\%). A acinesia matinal foi descrita, como única manifestação da flutuação motora, em $4,8 \%$ dos pacientes.

Com relação ao tipo de movimento, houve predomínio do movimento coreiforme, seguindose, em frequência, distonia, coreodistonia e discinesia orofacial (Tabela 2). Na Tabela 2 observa-se uma soma superior ao número total de pacientes da amostra devido o fato de ter sido descrito mais de um tipo de movimento anormal em um mesmo paciente em momentos diferentes do acompanhamento clínico. Tal fenômeno foi descrito em $34,7 \%$ dos casos revisados. $\mathrm{O}$ movimento coreiforme foi descrito com maior frequência na forma generalizada $(43,2 \%$ dos pacientes com movimento coreiforme), na face $(26,3 \%)$ e nos membros superiores $(12,7 \%)$. Observou-se predomínio da distonia nos membros inferiores ( $44,9 \%$ dos pacientes com distonia). A distonia matinal ocorreu em 24 pacientes, correspondendo a $35 \%$ do total de casos de distonia. Como única manifestação discinética, foi descrita em apenas 7 pacientes.

A discinesia ocorreu de forma unilateral em 38,6\% dos pacientes. Nestes pacientes os movimentos anormais ocorreram mais frequentemente no hemicorpo mais comprometido pela DP $(63,2 \%)$.

Quanto ao tipo de discinesia, a mais frequentemente descrita foi a de "pico de dose", seguindose a discinesia tipo "contínua" e "bifásica". Em 6 pacientes não foi possível classificar o tipo de discinesia. Mais uma vez, observamos soma de cventos superior ao total de pacientes da amostra pelo fato de vários pacientes terem apresentado mais de um tipo de discinesia (Tabela 1). As discinesias do tipo

Tabela 2. Frequência dos tipos de movimentos anormais apresentados pelo pacientes parkinsonianos com discinesias associadas com o uso de levodopa.

\begin{tabular}{ccc}
\hline Tipo de movimento & Frequência absoluta (n) & Frequência relativa (\%) \\
\hline Coreiforme & 87 & 40,5 \\
Distonia & 69 & 32,1 \\
Coreodistonia & 28 & 13,0 \\
Dicinesia orofacial & 31 & 14,4 \\
Total & 215 & 100 \\
\hline
\end{tabular}

(n) superior ao total da amostra devido a sobreposição de eventos. 
"pico de dose" e "contínua" assaciaram-se com movimentos coreiformes em 46,2 e 50\% dos casos, respectivamente, e a discinesia do tipo "fim de dose" com distonia na maior parte das vezes (96,5\%).

A média de idade de início da DP dos pacientes com discinesia orofacial isolada foi 66,2 anos. A daqueles que apresentavam a discinesia orofacial associada a outro movimento anormal foi 52,2 anos e a daqueles que não a apresentavam foi 51,3 . A diferença é significante de acordo com o teste de Kruskal-Wallis (H calc $=12,59 ; \mathrm{H}$ crítico $=5,99$ ).

\section{COMENTÁRIOS}

As discinesias são uma complicação frequente nos pacientes parkinsonianos tratados com levodopa. A frequência desta complicaçāo varia nos diversos estudos já realizados, com metodologias diferentes, e oscila entre $18 \%$ até $91 \%$ nos pacientes tratados com levodopa por mais de 5 anos ${ }^{5.10}$.

Considerando a idade de início da DP nos pacientes que desenvolveram discinesias, observamos a média de 52,5 anos. Vários autores consideram que os pacientes com idade de início da DP abaixo dos 60 anos parecem ser mais susceptiveis ao desenvolvimento das discinesias ${ }^{9.16}$. Em estudo pareado, para tempo de tratamento com levodopa e gravidade da doença, com pacientes parkinsonianos mais jovens e idosos, Gibb \& Lees observaram que $91 \%$ dos pacientes jovens apresentavam discinesias relacionadas a levodopa contra $69 \%$ dos idosos ${ }^{10}$.

Existe a hipótese de que nos pacientes parkinsonianos, com idade de início mais precoce da DP, as alteraçōes no SNC concentram-se principalmente no sistema dopaminérgico, enquanto nos pacientes mais idosos o comprometimento é mais difuso e envolva outros sistemas neuromediadores, como o serotoninérgico e gabaérgico. A deficiência dopaminérgica predominante levaria a uma supersensibilização dos receptores dopaminérgicos, aumentando a susceptibilidade às discinesias ${ }^{11}$.

O tempo de uso de levodopa e a duraçāo da doença de Parkinson no início das discinesias desta amostra se assemelham àqueles encontrados na literatura ${ }^{6,12}$. Há autores que questionam a importância do tempo de uso de levodopa no aparecimento das discinesias, já que em suas amostras as discinesias surgiram no decorrer do primeiro ano de tratamento com a levodopa na maioria dos pacientes ( $61 \%$ na amostra de Friedman e $72 \%$ na de Bergmann e col.) $)^{4,9}$.

As discinesias ocorreram preferencialmente em pacientes que se achavam nos estágios II e III de Hoehn \& Yahr, e os pacientes que iniciaram o tratamento com levodopa nos estágios mais avançados desenvolveram as discinesias mais precocemente. É possível que o grau de comprometimento clínico dos pacientes no início do tratamento com levodopa tenha influenciado o aparecimento das discinesias mais do que o tempo de tratamento com levodopa ou a duração da DP como um todo. Portanto, parece que qưanto mais rapidamente evolui a doença, maior é a possibilidade de surgirem as discinesias $^{1,4,5}$.

Com relaçāo a ocorrência de flutuação motora nos pacientes com discinesias, os autores concordam que esta associaçāo é frequente e representa a complicação mais comum do tratamento com levodopa a longo prazo ${ }^{3,4,24}$. Bergmann e col. afirmam não haver correlação significante entre o tempo até o aparecimento das discinesias e o tempo que o paciente leva para apresentar as flutuaçōes motoras ${ }^{4}$.

O predomínio dos movimentos anormais no mesmo lado onde havia predomínio dos sinais ou sintomas da DP é achado comum nos estudos já realizados sobre a discinesia ${ }^{12,18,25,30}$. Segundo Horstink, este achado reforça a hipótese de que o grau de comprometimento clínico é fator relevante na gênese das discinesias, ou seja, as discinesias surgiriam mais frequentemente no hemicorpo mais afetado pela denervação dopaminérgica e, portanto, de maior gravidade da DP ${ }^{12}$.

O movimento do tipo coreiforme, o mais frequentemente observado, prevaleceu na forma generalizada e na face, sendo pouco frequente nos membros inferiores. Em nossa amostra de pacientes a frequência da discinesia orofacial está abaixo da frequência habitualmente observada na maioria dos estudos clínicos revisados, em que a discinesia com localização no segmento cefálico é considerada como a mais frequente e a primeira que surge quando o paciente com DP desenvolve as 
discinesias ${ }^{18,19,21,23,27,30}$. É possível que esta diferença se deva ao fato de não termos considerado a discinesia orofacial quando esta fazia parte do quadro coreiforme generalizado, mas apenas quando surgiu isoladamente.

A discinesia apresentou-se na forma de distonia em $40 \%$ da nossa casuística, semelhante aos percentuais descritos por Kindron \& Melamed, que observaram distonia em 47\% de 207 pacientes avaliados, e por outros autores ${ }^{13.222 .26,27}$. A distonia predominou nos membros inferiores e, no caso específico da distonia matinal, todos os pacientes apresentaram o quadro distônico nos membros inferiores. O percentual de distonia matinal na nossa amostra foi semelhante ao relatado por Poewe \& Lees, que descrevem a distonia matinal em um terço dos pacientes com distonia ${ }^{29}$. Kindron \& Melamed referem-se à distonia matinal como a forma mais frequente de distonia nos pacientes parkinsonianos em tratamento com a levodopa ${ }^{13}$.

Observamos maior tendência da discinesia orofacial ocorrer como única manifestação discinética nos pacientes mais idosos, tendência esta já observada por outros autores ${ }^{8.22}$. Fahn afirma que a discinesia orofacial é o movimento involuntário que mais ocorre e que, quando surge isoladamente, é mais frequentemente visto em pacientes mais idosos ${ }^{8}$.

Luquin e col. descrevem um padrão de localizaçāo dos movimentos discinéticos relacionados ao tipo de discinesia e sugerem a existência de uma predileção somatotópica para a expressão clínica das discinesias, o que explicaria porque os quadros distônicos tendem a predominar nos membros inferiores e os coreiformes nos membros superiores $\mathrm{e}$ face ${ }^{18}$. Outros atribuem este padrāo de localização das discinesias à organizaçāo somatotópica e neuroquímica do núcleo caudado e do putâmen ${ }^{14.22}$. Segundo a hipótese de Marconi e col., ocorreria um gradiente de excitação dos receptores dopaminérgicos estriatais. Inicialmente seriam estimulados os receptores $\mathrm{D}_{2}$ da região dorsal do putamen, mais sensíveis devido a maior denervação nesta região e, portanto, estimulados mesmo com baixos niveis de dopamina. Com o aumento progressivo do nível de dopamina seriam estimulados os receptores da regiāo ventral, onde predominam receptores $D_{1}$. Segundo os autores isto explicaria a tendência de ocorrer movimentos distônicos (pela estimulação de $\mathrm{D}_{2}$ ) nos membros inferiores em situação de baixo nível de dopamina e de movimentos coreiformes (pela estimulaçāo de $\mathrm{D}_{1}$ ) na extremidade superior do corpo em situação de alto nível de dopamina. Os mecanismos farmacodinâmicos responsáveis por este gradiente nāo sāo claros. A hipótese apoia-se no fato das projeçōes cortico-estriadas provenientes do território correspondente aos membros inferiores dirigirem-se à parte dorsal do putamen, $e$ as dos membros superiores, tronco e face à parte ventral ${ }^{16}$.

Considerando o exposto, acreditamos que o grau de comprometimento clínico do paciente seja um dos fatores críticos na facilitação para o aparecimento das discinesias nos pacientes parkinsonianos tratados com levodopa, por mecanismos ainda não completamente elucidados. No entanto, novos estudos, prospectivos e controlados, são necessários para avaliar efetivamente o papel do grau de comprometimento clínico na gênese das discinesias induzidas pela levodopa.

Agradecimentos - Agradecemos a CAPES pelo financiamento parcial deste estudo e ao Prof. Neil F. Novo (Departamento de Bioestatística da Escola Paulista de Medicina) pelo auxílio na análise estatística.

\section{REFERÊNCIAS}

1. Ballard PA, Tetrud JW, Langston JW. Permanent human parkinsonism due to 1-methyl-4-phenyl-1,2,3,6tetra-hydropyridine (MPTP): seven cases. Neurology 1985, 35:949-956.

2. Barbeau A. L-dopa therapy in Parkinson's disease: a critical review of nine years' experience. Can Med Assoc J 1969, 27:59-68.

3. Barbeau A. Parkinson's disease: clinical features and etiopathology. In Vinken PJ, Bruyn GW, Klawans HL (eds). Handbook of Clinical Neurology , Vol. 49: (Extrapyramidal disorders). New York: Elsevier, 1986, p87-152.

4. Bergmann KJ, Mendoza MR, Yahr MD. Parkinson's disease and long-term levodopa therapy. Adv Neurol 1986, 45:463-467.

5. Birkmayer W, Hornykiewicz O. Der 1-3,4-dihidroxyphenylalanin (DOPA): Effekt bei der Parkinson-Akinese. 
Wien Klin Wschr 1961, 73:787-788.

6. Blin J, Bonnet A, Agid Y. Does levodopa aggravate Parkinson's disease? Neurology 1988, 38:1410-1416.

7. Cotzias GC, Van Woert MH, Schiffer LM. Aromatic amino acids and modification of parkinsonism. $N$ Engl J Med 1967, 276:374-379.

8. Fahn S, Bressman SB. Should levodopatherapy for parkinsonism be started early or late? Evidence against early treatment. Can J Neurol Sci 1984, 11:200-206.

9. Friedman A. Levodopa-induced dyskinesia: clinical observations. J Neurol 1985, 232:29-31.

10. Gibb WRG, Lees AJ. A comparison of clinical and pathological features of young and old-onset Parkinson's disease. Neurology 1988, 38:1402-1406.

11. Granérus AK, Carlsson A, Svanborg A. The aging neuron: influence on symptomatology and therapeutic response in Parkinson's syndrome. Adv Neurol 1979, 24:327-334.

12. Horstink MWIM, Zijlmans JCM, Pasman HJ, Berger HJC, Van't Hof MA. Severity of Parkinson's disease is a risk factor for peak-dose dyskinesia. J Neurol Neurosurg Psychiatry 1990, 53:224-226.

13. Kidron D, Melamed E. Forms of dystonia in patients with Parkinson's disease. Neurology 1987, 37:1009-1011.

14. Kish S, Rajput A, Gilbert J. Distribuition of striatal GABA in normal and parkinsonian brain [Abstr]. New York: VIII International Symposium on Parkinson's disease, 1985.

15. Kish SJ, Shannak HK, Hornykiewicz O. Uneven pattern of dopamine loss in the striatum of patients with idiopathic Parkinson's disease: pathophysiologic and clinical implications. N Engl J Med 1988, 318:876-880.

16. Kostic V, Przedborski S, Flaster E, Sternic N. Early development of levodopa-induced dyskinesias and response fluctuations in young-onset Parkinson's disease. Neurology 1991, 41:202-205.

17. Künzle $\mathrm{H}$. Bilateral projections from precentral motor cortex to the putamen and others parts of the basal ganglia: an autoradiographic study in Maccaca fascicularis. Brain Res 1975, 88:195-209.

18. Luquin MR, Scipioni O, Vaamonde J, Gershanik O, Obeso JA. Levodopa-induced dyskinesias in Parkinson's disease: clinical and pharmacological classification. Mov Disord 1992, 7:117-124.

19. Markham CH. The choreoathetoid movement disorder induced by levodopa. Clin Pharmacol Ther 1971, 12:340-346.

20. Marconi R, Lefebvre-Caparros D, Bonnet AM, Vidailhet M, Dubois B, Agid Y. Levodopa-induced dyskinesias in Parkinson's disease - phenomenology and pathophysiology. Mov Disord 1994, 9:2-12.

21. Marsden CD, Parkes JD, Quinn N. Fluctuactions of disability in Parkinson's disease: clinical aspects. In Marsden CD, Fahn S. (eds) Neurology 2: movement disorders. London: Butterworths, 1982, p. 96-122.

22. Melamed E. Early-morning dystonia. Arch Neurol 1979, 36:308-310.

23. Mones RJ, Elizan TS, Siegel GJ. Analysis of L-dopa induced dyskinesias in 51 patients with parkinsonism. J Neurol Neurosurg Psychiatry 1971, 34:668-673.

24. Mouradian MM, Heuser JE, Baronti F, Fabbrini G, Juncos JL, Chase TN. Pathogenesis of dyskinesias in Parkinson's disease. Ann Neurol 1989, 25:523-526.

25. Narabayashi H, Yokochi F, Nakajima Y. Levodopa-induced dyskinesia and thalamotomy. J Neurol Neurosurg Psychiatry 1984, 47:831-839.

26. Nausieda PA, Weiner WJ, Klawans HL. Dystonic foot response of parkinsonism. Arch Neurol 1980, 37:132136.

27. Nutt JG. Levodopa-induced dyskinesia: review, observations, and speculations. Neurology 1990 , 40:340-345.

28. Papavasiliou PS, Cotzias GC, Duby SE. Levodopa in parkinsonism: potentiation of central effects with a peripheral inhibitor. N Engl J Med 1972, 285:8-14.

29. Poewe WH, Lees AJ. The pharmacology of foot dystonia in parkinsonism. Clin Neuropharmacol 1987, 10:47-56.

30. Tolosa E, Alom J, Marti MJ. Drug induced dyskinesias. In Jankovic J, Tolosa E (eds).- Parkinson's disease and movement disorders. Ed 2. Baltimore: Williams \& Wilkins, 1993, p.375-397. 